\title{
Two Cases of Dyskeratosis Congenita with Clinically Distinct Presentations, Seen in National University Hospital, Singapore
}

\author{
Lester Juay Nisha Suyien Chandran \\ Department of Dermatology, National University Hospital, Singapore, Singapore
}

\section{Established Facts}

- Dyskeratosis congenita (DKC) is a genodermatosis of variable inheritance and is a congenital telomeric disorder with multisystemic manifestations.

- DKC has high morbidity and mortality, particularly from haematological complications if left undetected and untreated in the early stages.

\section{Novel Insights}

- The classical mucocutaneous triad of reticulated hyperpigmentation, nail dystrophy, and leukoplakia may not however be present in all patients with $\mathrm{DKC}$, leading to delays in diagnosis.

- Dermatologists should hold a high index of suspicion for DKC in young patients who present without the classical triad of features, as early dermatological care can be instituted through reinforcement of rigorous sun protection and regular surveillance for skin cancers. Early detection also allows for time to plan for haematopoietic stem cell transplantation, which may dramatically improve the survival of these patients, as bone marrow failure is often inevitable.

\section{Keywords}

Genodermatoses · Genetic skin disorders · Pigmentary disorders

\section{Abstract \\ Dyskeratosis congenita (DKC) is a genodermatosis of vari- able inheritance and is often characterised by the classical triad of nail dysplasia, reticulate hyperpigmentation of up- per chest and neck, and oral leukoplakia. We report 2 cases of DKC from National University Hospital, Singapore, whose clinical presentations differed greatly from each other. Der-}

matologists should hold a high index of suspicion for DKC in young patients who present without the classical triad of features, as early dermatological care can be instituted through reinforcement of rigorous sun protection and regular surveillance for skin cancers. Early diagnosis also offers physicians the time to organise haematopoietic stem cell transplantation if necessary, as bone marrow failure is often inevitable. As a multisystemic disease with high morbidity and mortality particularly from haematological complications if left undetected and untreated in the early stages, the role of the dermatologist in diagnosing DKC is a crucial one.

(C) 2021 The Author(s)

Published by S. Karger AG, Basel
(C) 2021 The Author(s)

Published by S. Karger AG, Basel

This article is licensed under the Creative Commons Attribution 4.0 International License (CC BY) (http://www.karger.com/Services/ OpenAccessLicense). Usage, derivative works and distribution are permitted provided that proper credit is given to the author and the original publisher.
Correspondence to:

Lester Juay, lester_juay@nuhs.edu.sg 


\section{Introduction/Literature Review}

Dyskeratosis congenita (DKC) is a congenital telomeric disorder with multisystemic manifestations [1]. While $\mathrm{DKC}$ is a monogenic disorder, its inheritance pattern is variable, with 11 genes being implicated thus far [2]. While common presentations are usually localised to skin, mucous membranes, and nails, DKC is associated with progressive bone marrow failure and increased cancer risks, particularly of myeloid leukaemia and head and neck squamous carcinomas [3]. The classical mucocutaneous triad of reticulated hyperpigmentation, nail dystrophy, and leukoplakia may not however be present in all patients with $\mathrm{DKC}$, leading to delays in diagnosis. We share 2 cases of DKC seen in National University Hospital, Singapore, whose clinical presentations differ greatly from each other.

\section{Case Reports}

The first case was an 18-year-old Indian gentleman, who first presented to the dermatologist for loss of fingerprints. This was brought to attention by the immigration authorities when he attempted to travel abroad. Dermatological examination revealed generalised mottled and confetti-like hypopigmentation over his trunk and limbs, dystrophic nails, and reticulate hyperpigmenta- tion and white plaques on the oral mucosa (Fig. 1). Initial differentials considered were reticulate acropigmentation of Kitamura, dyschromatosis symmetrica hereditaria, Naegeli-FranceschettiJadassohn syndrome, dermatopathia pigmentosa reticularis, and Dowling-Degos disease. Mucosal lichen planus would be a differential for the oral lesions; however, the other cutaneous findings were inconsistent. Punch biopsy and genetic testing were offered but initially declined.

Further history was obtained on reviews: he was diagnosed with presumed immune thrombocytopaenic purpura at the age of 11, with resultant steroid-induced avascular necrosis of bilateral hips. Clinical suspicion of DKC was made by the dermatologist; genetic testing subsequently revealed TINF2 gene mutation, confirming the diagnosis of autosomal dominant DKC. Despite the inheritance pattern of this gene, his parents and siblings were tested negative for the same point mutation in the TINF2 gene, suggesting a germline or post-zygotic mutation. He progressed to bone marrow failure within 3 years following diagnosis, necessitating allogeneic haematopoietic stem cell transplant. The first transplant eventually failed from graft rejection, but the second haploidentical stem cell transplant eventually led to bone marrow reconstitution. Unfortunately, he passed away 2 years after transplant from a ruptured hepatic angiosarcoma. The mucocutaneous changes remained largely stable in the annual clinic review after the transplant, prior to his demise.

The second case was a 7-year-old Chinese boy who presented initially to the otolaryngologist for recurrent tongue ulcers since the age of 3 . A dentist subsequently noted dysplastic teeth and oral leukoplakia (Fig. 2), who then referred the patient to a geneticist for concerns of DKC. The geneticist elicited a strong family history of males on the maternal side dying young from marrow fail-

Fig. 1. a, b Generalised mottled and confetti-like hypopigmented macules, best appreciated over the trunk. c Typical nail changes of onychoschizia and longitudinal ridging are well appreciated here. d Reticulated hyperpigmented streaks are seen on the buccal and lingual surfaces. The white plaques of leukoplakia are subtle and are not well visualised in this photo.

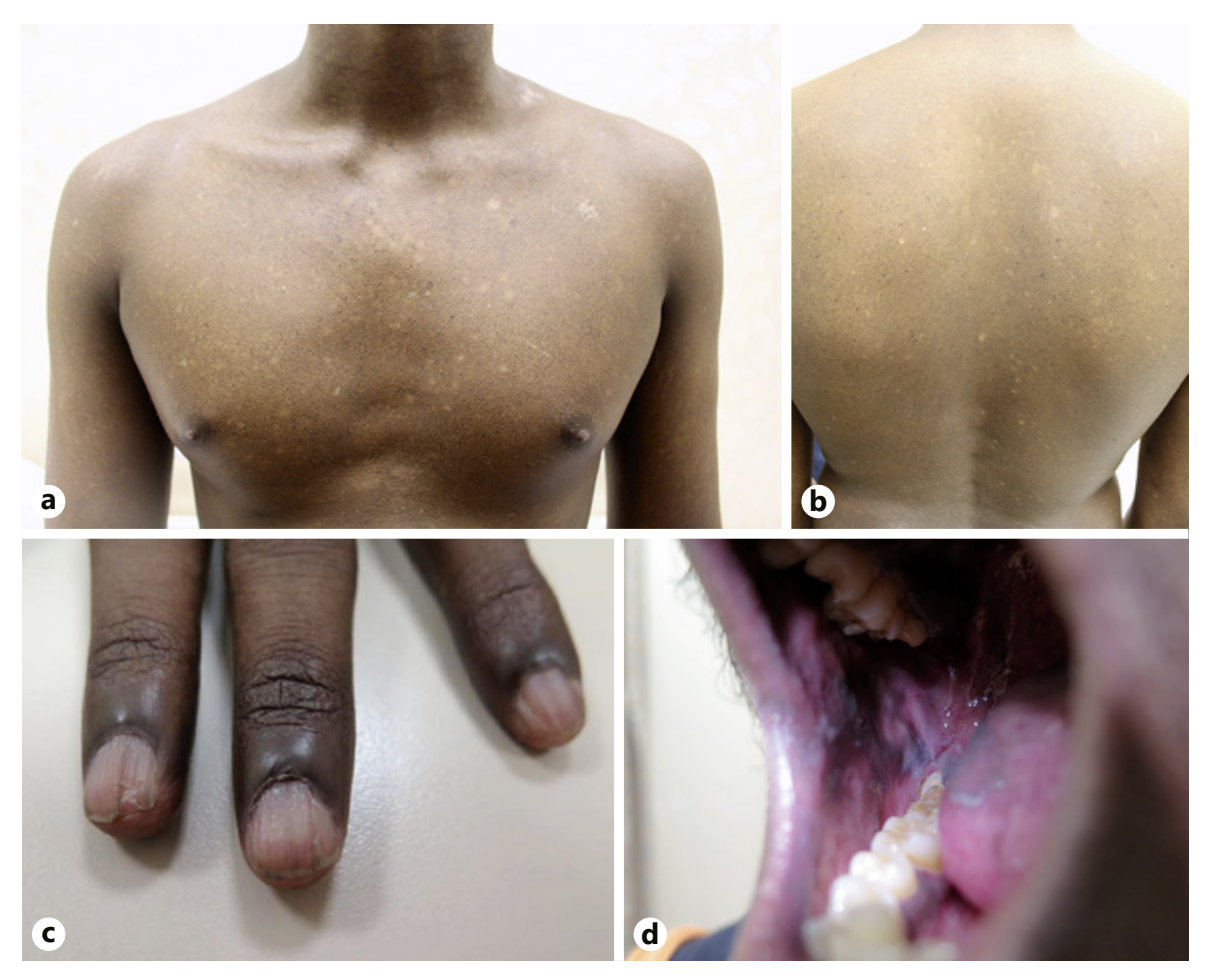


ure. Genetic testing revealed a hemizygous pathogenic DKC1 variant on the X-chromosome, confirming the diagnosis of X-linked DKC. Subsequent dermatology review revealed reticulate hyperpigmentation of the buccal mucosae, large white plaques on the dorsal tongue, with only subtle reticulate hyperpigmentation of the anterior neck crease, and no nail changes (Fig. 2). He has since been on regular follow-up with the dermatologist and haematologist and remains well at the point of last assessment.

\section{Discussion}

In the first case, our patient was found to have TINF2 mutation. TINF2 mutations tend to lead to severe disease, with most developing aplastic anaemia by the age of 10 years [4]. Analysis of a cohort of genetically uncharacterised patients from the Dyskeratosis Congenita Registry in the UK revealed a high prevalence and early presentation of aplastic anaemia in patients with TINF2 mutations, in contrast to those without the mutation. This suggests that screening for mutations in TINF2 could prove valuable in the subgroup of DKC patients with sporadic presentation without a family history. In the second case, the young boy presented with dysplastic teeth, which was not a feature within the classic triad. There have been many cases of dental changes documented in patients with DKC, which include decreased root/crown ratio, taurodontism, hypodontia, and extensive tooth decay [5]. Irregular mucosal ulcers, which were seen in our patient, have been reported as mucosal signs of DKC [6]. Hence, a complete evaluation of young patients with incomplete mucocutaneous signs should entail a dental examination. In this case, it was fortuitous that the dentist noticed the dental
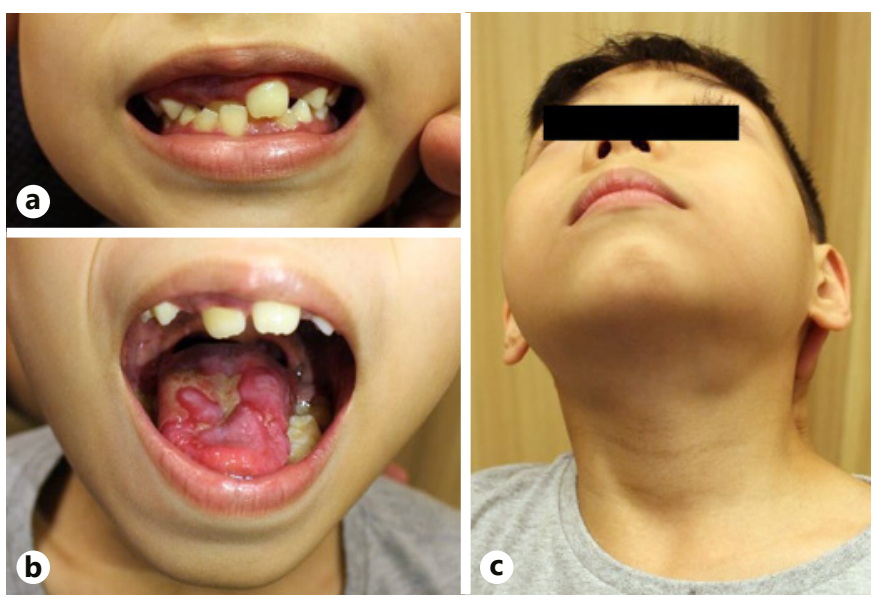

Fig. 2. a, b The dysplastic teeth and geographic white plaques on the tongue were first noticed by the dentist. c Subtle reticulate hyperpigmentation of the anterior neck crease can be seen. dysplasia and oral leukoplakia and promptly referred to the dermatologist upon suspicion of DKC.

Dermatologists should hold a high index of suspicion for DKC in young patients who present without the classical triad, as this genodermatosis portends high morbidity and mortality. Bone marrow failure accounts for $70 \%$ of all mortality, with up to $15 \%$ of deaths caused by pulmonary complications and $10 \%$ by malignancy [7]. Pulmonary diseases include pulmonary fibrosis, or in severe cases, fatal pneumothoraces [8]. Solid organ cancers, such as head and neck squamous cell carcinomas, skin cancers, and anorectal carcinomas [9], have an aggressive natural history in patients with $\mathrm{DKC}$, as highlighted in a recent case report [10]. Early dermatological care can be instituted through reinforcement of rigorous sun protection and regular skin cancer surveillance. Early diagnosis of $\mathrm{DKC}$ also allows for time to plan for haematopoietic stem cell transplantation, which may dramatically improve the survival of these patients, as bone marrow failure is often an inevitable sequela in the natural history. Smoking cessation and abstinence from alcohol are also key, as they compound the already high risk of oral cancers in patients with DKC [11]. The role of the dermatologist in diagnosing DKC is a crucial one, and further management needs to be holistic, through multidisciplinary involvement of the haematologist and otolaryngologist.

\section{Acknowledgment}

The authors are grateful for the patients and their families, who have so graciously offered their photos and their stories for the betterment of medical science.

\section{Statement of Ethics}

Ethical approval was not required for publishing of case reports fewer than 3 cases, as stated by the Singapore Institutional Review Boards. Written informed consent was obtained from the parents of both subjects for publication of the details of their medical case and any accompanying images.

\section{Conflict of Interest Statement}

The authors have no conflicts of interest to declare.

\section{Funding Sources}

No funding was needed for preparation of the manuscript. 


\section{Author Contributions}

Lester Juay (corresponding author) performed the literature review, wrote the manuscript, and selected photos for the manuscript. Nisha Suyien Chandran (co-author) provided both cases and oversaw multiple revisions of the final manuscript.

\section{Data Availability Statement}

All data generated or analysed during this study are included in this article. Further enquiries can be directed to the corresponding author.

\section{References}

1 Kirwan M, Dokal I. Dyskeratosis congenita: a genetic disorder of many faces. Clin Genet. 2008;73(2):103-12.

2 Savage SA, Dokal I, Armanios M, Aubert G, Cowen EW, Domingo DL, et al. Dyskeratosis congenita: the first NIH clinical research workshop. Pediatr Blood Cancer. 2009;53(2): 520-3.

3 Alter BP, Giri N, Savage SA, Rosenberg PS. Cancer in dyskeratosis congenita. Blood. 2009;113(26):6549-57.

4 Walne AJ, Vulliamy T, Beswick R, Kirwan M, Dokal I. TINF2 mutations result in very short telomeres: analysis of a large cohort of pa- tients with dyskeratosis congenita and related bone marrow failure syndromes. Blood. 2008; 112(9):3594-600.

5 Serindere G. Oral and dental changes of dyskeratosis congenita: a case report with literature review. J Adv Oral Res. 2018;9(1-2):20-3.

6 Baran I, Nalcaci R, Kocak M. Dyskeratosis congenita: clinical report and review of the literature. Int J Dent Hyg. 2010;8(1):68-74.

7 Dokal I. Dyskeratosis congenita. Hematol Am Soc Hematol Educ Program. 2011;2011(1): 480-6.

8 Boueiz A, Abougergi MS, Noujeim C, Assaf EB, Jamaleddine G. Fatal bilateral pneumo- thoraces complicating dyskeratosis congenita: a case report. J Med Case Rep. 2009;3:6622.

9 Alter BP, Giri N, Savage SA, Rosenberg PS. Cancer in dyskeratosis congenita. Blood. 2009;113(26):6549-57.

10 Manfuso A, Risitano AM, Copelli C. Dyskeratosis congenita and squamous cell carcinoma of the mandibular alveolar ridge. BMJ Case Rep. 2021;14(5):e242459.

11 Pelucchi C, Gallus S, Garavello W, Bosetti C, La Vecchia C. Cancer risk associated with alcohol and tobacco use: focus on upper aerodigestive tract and liver. Alcohol Res Health. 2006;29(3):193-8. 